scarcity of documentation is perforce out of all relation to their importance to the marine ecologist. This is no fault of Dr Lauckner's but it does impede the objective of helping biologists to give disease its appropriate place within the marine ecological model.

Since very few of the invertebrates covered in this volume are cultured, or even fished, the economic incentive for the detailed scientific study of their diseases has been absent. Most of the records of such diseases are one-off observations rather than systematic studies and often even Dr Lauckner is unable to do more than detail the paucity of available information.

Although the book is well produced and beautifully printed, most pathologists (though not, I suspect, parasitologists) would jib at the extensive use of line drawings rather than photomicrographs in all but a very limited number of instances.

The gathering together of such a mass of information, often related only by its ecosystem, and its synthesis into a review of this magnitude indicates great determination. As a source of information for scholars of a wide range of subjects it will be invaluable. I doubt, however, if it will substitute for a specific monograph on any of the groups covered. It is neither specialized enough nor complete. I do expect, though, that the complete series will find its place in any library attempting to cover the field of marine biology.

R.J. Roberts is Director of the Institute of Aquaculture at the University of Stirling, UK.

\section{An 'axiomatic' approach to quantum mechanics}

\section{J.L. Martin}

Quantum Mechanics. By A. Bohm. Pp. 522. (Springer: Heidelberg and New York, 1979.) DM 58, \$31.90.

IN WRITING about quantum mechanics, where does an author begin? There are several possibilities. He may struggle through the historical development of the subject, with all its gropings and false starts - excellent in its place, but not in a standard text. He may begin with elementary physical examples, and using a judicious mixture of assertion, intuition, correspondence principle and Ehrenfest's theorem - uncluttered with too much mathematics - attempt to lead the reader into a 'feel' for the subject. Or he may adopt an 'axiomatic' approach: 'Here are the rules; where do they lead?'. The trouble with this last option is that the reader who is looking for enlightenment may have to journey for too long in the dark before the dawn of what it is all about.

This is an 'axiomatic' book, and I would hazard the opinion that it is not for the physicist - not even for the theoretical physicist - but for the mathematician who is already familiar with the basic structure of quantum mechanics. Moreover, the selection of topics obviously reflects the author's personal interests to a degree; thus I find it difficult to recommend the book as a text, as the coverage is so uneven. It may well be useful to anyone with an interest in the mathematical aspects of quantum mechanics, developed from a particularly algebraic point of view. (The very first mention of a physical system, p.10, runs: "The mathematical image of a physical system is an operator *-algebra in a linear scalar-product space $\mathscr{H}$ ". This sets the tone of the book as a whole.) The reader who wishes to learn to use quantum mechanics as a tool will be disappointed; a glance at the problems at the end of each chapter will confirm this. Compare them, for example, with those in classics such as Schiff
(Quantum Mechanics. McGraw-Hill: New York, 1968).

In reading the book, I felt I was listening to the lectures. The presentation in parts was too discursive and rambling for my taste: after all, the reader can re-read the text if he needs to, and writing a book is a golden opportunity to tighten up a lecture style into something more terse and tidy, in which the wood can be seen in spite of the trees.

There are many small and usually unimportant misprints which betray a lack of care at the proof-reading stage "communication relations" (p.41); in the index under "Selection rules", "hydrogen bomb" should surely be "hydrogen atom". And by the way, the Argand diagram was not introduced to help us understand the scattering amplitude. Jean Robert Argand did his work in 1806!

J.L. Martin is Reader in Physics at King's College, University of London, UK.

\section{Controversial view of primate evolution}

\section{R. D. Martin \\ Evolutionary History of the Primates. By Frederick S. Szalay and Eric Delson. Pp.580. (Academic: New York and London, 1980.) \$48, £31.20.}

THERE has been a great proliferation of research into the evolutionary radiation of the primates over the past 15 years. Reanalysis of the evidence has benefited from new palaeontological work, from a virtual explosion of new information on living primates (notably including biochemical data) and, above all, from reassessment of the methods used to infer phylogenetic relationships. As primate palaeontologists, Frederick Szalay and Eric Delson have both made significant contributions in the first of these areas, and Evolutionary History of the Primates is accordingly an authoritative and competent guide to the great array of recognized primate fossils.

A brief introduction includes, among other things, a handy chronological catalogue of known primate fossil sites and a simple outline of primate molar tooth morphology. The methods used for inferring evolutionary relationships, which owe much to Hennig, are touched upon; but this all-important aspect should have been discussed in full detail, especially since the authors themselves admit to a divergence of opinion. The bulk of the book consists of a systematic survey of primate groups, with notes on extant forms and extensive, well-illustrated descriptions of the fossils. In this respect, the book will undoubtedly serve as a valuable reference source and its usefulness is enhanced by a selective bibliography and a glossary of terms. Numerous good-quality scaled photographs combine to make this the most comprehensive primate fossil guide available to date, and it is to be recommended to anyone with an interest in primate evolution.

A sharp distinction must be drawn, however, between the mere description of fossil primate material and its interpretation within a comprehensive evolutionary framework. A severe limitation of this book lies in the exclusion of virtually all information other than that derivable from teeth and bones. It is particularly surprising that there is barely a reference to the evolution of the primate brain, since endocasts are now available to document fundamental features in numerous fossil primate species. In their introduction the authors accept that the reliability of phylogenetic inferences increases with the scope of the data analysed, yet they ignore or even discount out-of-hand much relevant information (e.g. immunological data) derived from living primates. A proper synthesis of both palaeontological and neontological data must be achieved before maximal accuracy can be expected.

The rationale underlying the classificatory scheme used in the book is also inadequately explained. In places, new taxonomic groupings have been established as direct concomitants of the authors' personal hypotheses regarding evolutionary affinities, whereas elsewhere 